\title{
The Key Technology of Image Transmission Based on ZigBee Jiaqiong Gao ${ }^{1, a}$ and Zhongsheng Wang ${ }^{2}$ \\ ${ }^{1}$ Department of Computer Science, Sichuan Vocational and Technical College Sichuan Suining 629000 \\ ${ }^{2}$ School of Computer Science and Engineering, Xi'an Technology University Xi'an 710021, China a516719510@qq.com
}

Keywords: ZigBee; CMOS camera; Compression; Decompression

\begin{abstract}
ZigBee technology owns the short distance, low rate, low complexity transmission properties, when it was used to transfer a large amount of data like image, it should be processed firstly, and then transferred. This paper introduces each node hardware design and software program design of the system. And this paper makes a test, using CMOS camera to collect image and transfer through the ZigBee network based on the image compression and decompression technique. Through the experiment test, it can be able to get a clear image that the resolution is $320 * 240$ and the size is about $13 \mathrm{~KB}$. This paper makes a research on the key technology about image transmission detailed, which has a certain research significance and practical value for the big data's transmission using ZigBee network.
\end{abstract}

\section{Introduction}

At present, using GPRS/CDMA technology realizes the wireless transmission of the image However, in some special cases, the whole network would be broken down if the base station has problem. But ZigBee technology is a self-organizing network can solve the problem. ZigBee technology is a low-cost, low-consumption network, which applied in automatic control, medical monitoring, smarthome, etc. But it cannot transmit image and audio. For this matter, this paper based on the system of the community endowment, creates an image acquisition system based on ZigBee.

\section{The Design of System Node}

The implementation of this system mainly includes two parts: the upper PC and lower PC. The lower PC is used to collect and transmit data, the upper PC is used to receive and process data. The gathering audio data derive form the camera could be processed by ARM. When the data collection is completed, the wireless sensor network created by central node implements the data transission.

PTC08 is a serial port camera which can collect data, shoot control, data comparession, serial stransmission. Image acquisition by camera is a standard JPEG format, need to be processed by ARM, through compression and subcontract, then transmite. As shown in Fig. 1. 


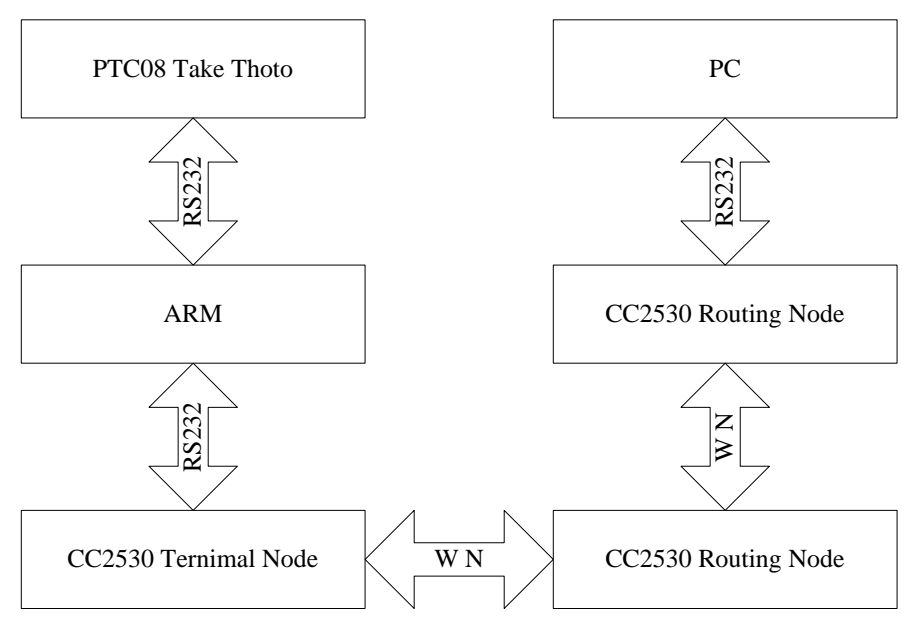

W N:Wireless Network

Figure 1. System flow chart figure

\section{The Design of Image Transmission System}

The system includes four parts: ZigBee data transmission module, ARM data transmission module, data processing module, and image data save and display module.

ZigBee Data Transmission Module. This module can realize the transparent data transmission, that is only the data format need be considered when the data in transit. And the lower PC is used to subpage and transmits data, the upper PC is used to receive and process data. The lower PC is connected to the PC by TS232 serial port, through the VC6.0++ MSCOMM control to receive serial data and processed by the upper PC's software.

ARM Data Transmission Module. This module includes four parts: device initialization, serial port setting, serial port communication, data processing. And it needs to ensure data integrity, security, accuray during transmission. The data transmission mechanism as follows: First, apply two buffers:buf1(a small buffer) and buf2(a large buffer).Using buf1 memory the send commands of upper PC, which need to receive and determine via serial port 0 , and the lower PC using serial port 1 receive it. Then, the lower PC using serial port 0 to send data if the data is smaller than a packet, or segmented in advance. To make the data correct, need to increase time-lag.

Processing Module. This module is a critical part to ensure the accuracy of data transmission. According to the characteristic of ZigBee network, set the packet size is 200B. The lower PC using serial port 0 to send data if the data is smaller than 200B,or segmented in advance. There exist relationship between packet size and transmission time. Therefore, modify the size of package based on the actual situation.

Data Save and Display Module. The PC is used to receive decompression, display and save data. If it is image save as .jpg format, else save as .txt format. Both might operate by click the Save data button and the Save Text data button. This system had the advantages of simple control, hign compression ratio, hign quality and hign performance and low price.

\section{The Definition of Data Frame}

In this system adopts the point-point communication means to transmit data between two nodes. Node, through judgment of the receive daa whether send to its owm, if so, then reveive, else drop.Frame format of send instruction: send command+data length+destination address+information, in which the data length is variable with a general length of 32 bytes. As shown in Fig. 2. 


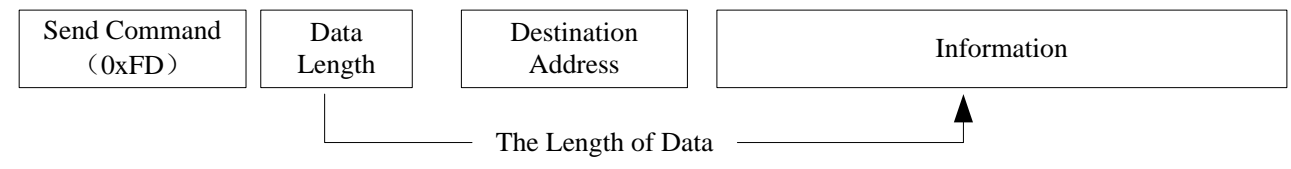

Figure 2. Send instruction format figure

Frame format of reveive instruction: send command + data length + destination address + information + source address. As shown in Fig. 3.

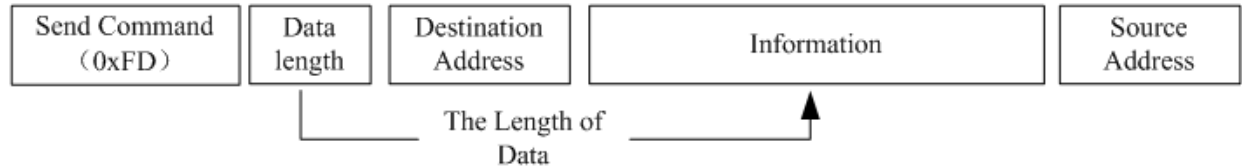

Figure 3. Receive data format figure

Set destination address to FFFF if the data send as a btoadcasting, and set destination address to 0000 if the data send to coordinator.

\section{Key Technology of Image Transmission}

The format of this system is a standard jpg, which had the advantages of simple control, hign compression ratio, hign quality and hign performance and low price.

The Process of Image Compression. During the process of image compression, First, the image is segmented into blocks of $8 * 8$ pixels; Second, forward dicrete cosine transform; Third, cause a two dimensional of AC and DC after quantify dicrete cosine transform(DCT); Fourth, Differential Pulse Code Modulation is used to encode AC and run length encoding is used to encode DC to improve compression efficiency; Fifth, get image data through query Huffman Tables. As shown in Fig. 4.

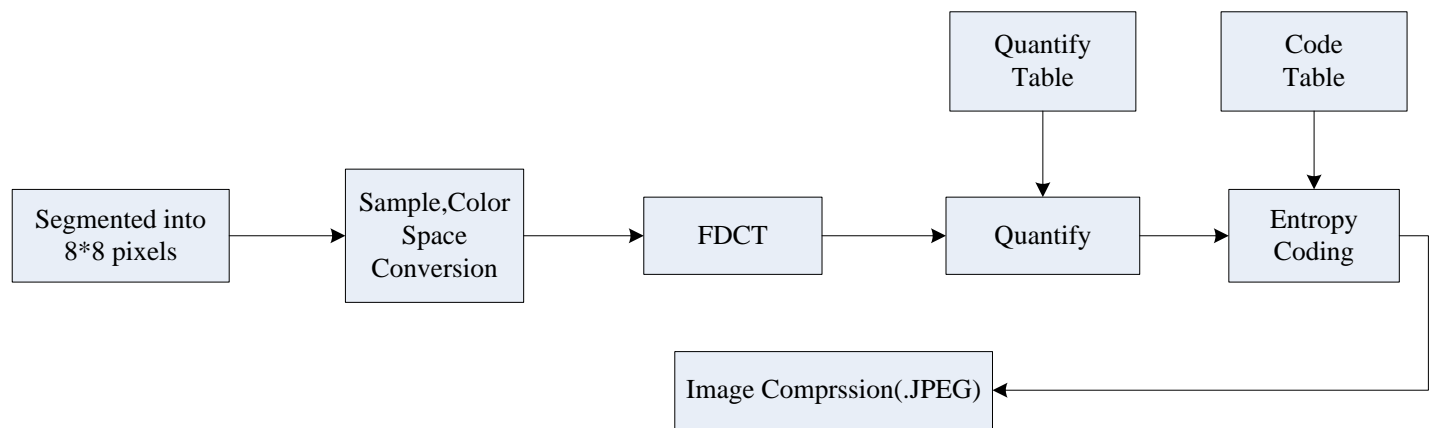

Figure 4. Image compression flowchart figure

The Process of Image Decompression. The process of image decompression includes a number of steps: One, extract information of file header. It includes information of file header and image data. Information of file header includes a lot of important information: version, length and width, Huffman Table, etc. Two, extract minimum code unit (MCU) and color component. Three, form $8 * 8$ data matrix through Huffman Table. Four, get a new $8 * 8$ data matrix trough differential encoding inverse quantization, anti-Zig-zag coding, inverse discrete consine transform. Five, convert $\mathrm{YcrCb}$ to RGB. Six, integrate data from every minimum code unit (MCU).

Note: during the process of coding, RGB minus 128 to meet the symmetry of domain, thus need to add 128. Operations defined as follows: 


$$
\begin{aligned}
& R=Y+1.402 \times C b+128 ; \\
& G=Y-0.34414 \times C r-0.71414 \times C b+128 ; \\
& B=Y+1.772 \times C b+128 ;
\end{aligned}
$$

During this process, if the value less than 0, then 0; if the value more than 255, than 255 .

\section{System Test}

This system can be able to get a clear image that the resolution is $320 * 240$ and the size is about 13 KB. As shown in Fig. 5 and Fig. 6.

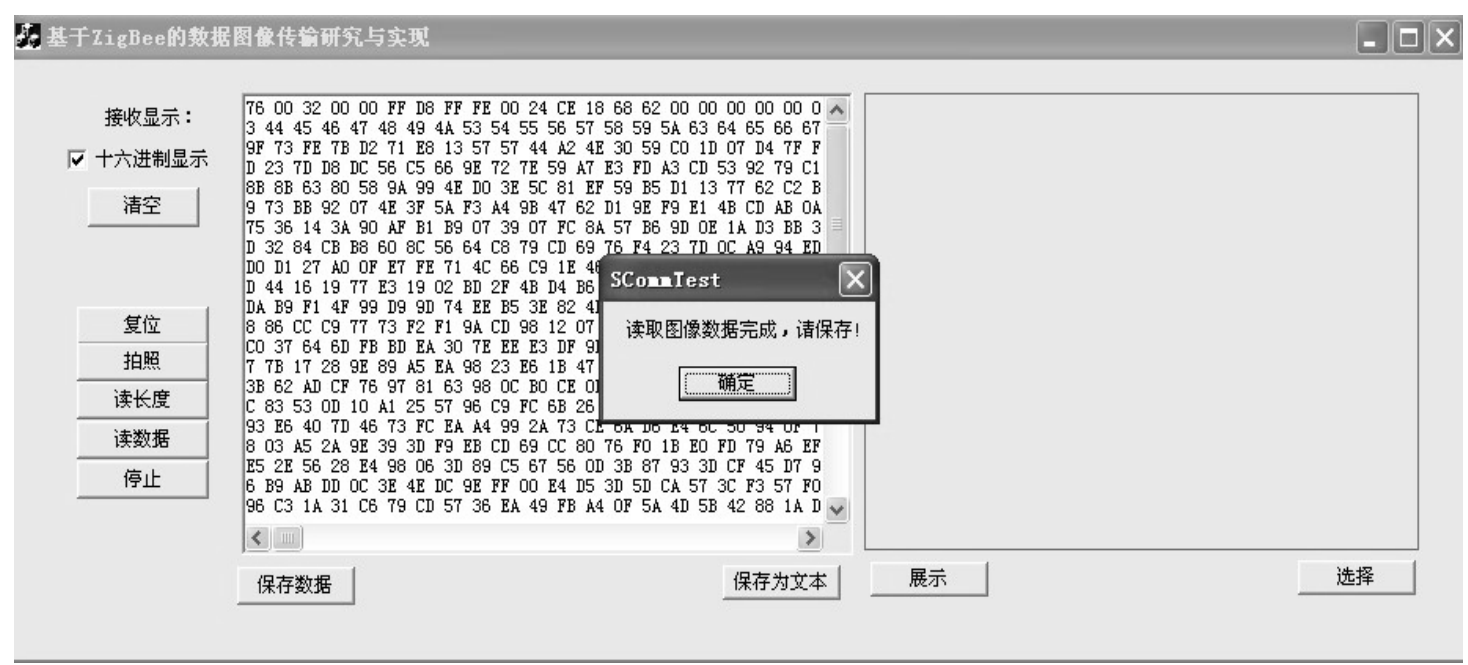

Figure 5. Image hexadecimal representation figure

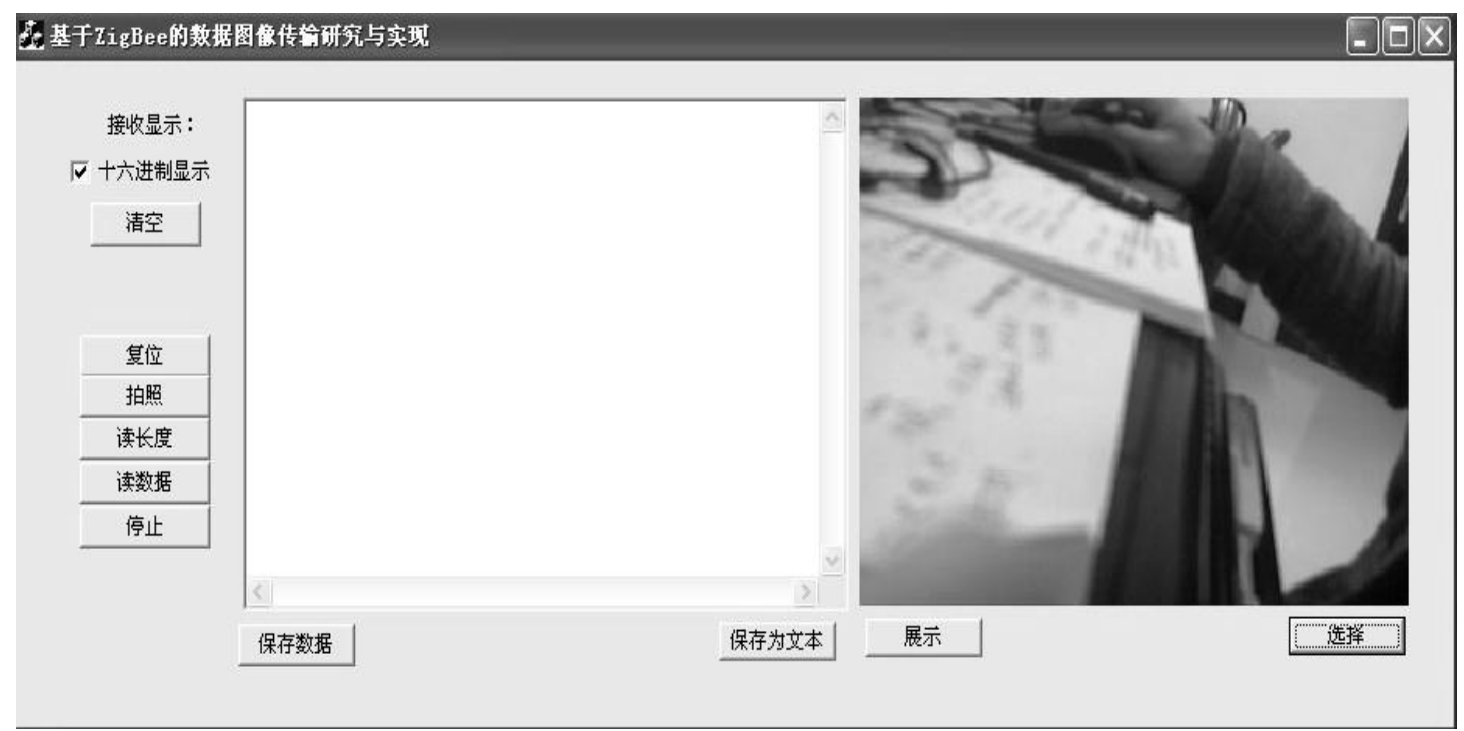

Figure 6. Image display figure

The hexadecimal image data show in Fig. 5 and save it by click the save data button. And the image data display in Fig. 6. Finally, realize the wireless transmission of the image data.

\section{Conclusions}

This paper introduces each node hardware design and software program design of the system, and realize the wireless transmission of the image data. Through a series of experiment and practice, this system can be able to get a clear image that the resolution is $320 * 240$ and the size is about 13 
KB. In the system of the community endowment help supervisor understand the state of old person. This paper has a certain research significance and practical value for the big data's transmission using ZigBee network.

\section{References}

[1] Z. Q. Song, X. Z. Zhou, and L. Wang, "Research on wireless image transmission based on ZigBee[J],” Computer Applications and Software vol. 29, no. 7, pp. 1-5, 2012.

[2] J. Lloret, I. Bosch, S. Sendra, and A. Serrano, "A wireless sensor network for vineyard monitoring that uses image processing[J]," Sensors, vol. 11, no. 6, pp. 6165-6196, 2014.

[3] L.Xiong, K.Y.Long. Design and Implementation of Wireless Network Based on ZigBee system [J].Computer Technology and Development, 2015.05, pp183-186, 2009.

[4] Z.Wang, D.L.Huang, Research on Key Technology of Image Transmission Based on ZigBee, Computer Technology and Development,2015.05,pp183-186.

[5] Zhongsheng Wang, Xiao Shang Research of AS level Network Topology, AJETR2015-02.

[6] Holman R, Stanley J, O zkan-Haller T.Applying video sensor networks to nearshore environment monitoring[J].IEEE Trans.on Pervasive Computing, 2003, 2(4);14-21.

[7] Akyildiz I F, Melodia T, Chowdhury K R. A survey on wireless multimedia sensor networks [J]. Computer Networks， 2007， PP921-925.

[8] S.Cheng,Q.M.shao,Q.ian.Image Transport Based on ZigBee Network, 2015.06 [J].Computer Engineering, PP68-71,2008.

[9] X.Q.Song, A New Image Compression Algorithm [J].Computer Engineering, 2006, 32(8) PP221-223. 